\title{
Genetic differentiation in marine and estuarine natural populations of Crassostrea angulata
}

\author{
Sagrario R. Michinina, Laureana Rebordinos* \\ Laboratorio de Genética, Facultad de Ciencias del Mar, Universidad de Cádiz, Polígono del Río San Pedro, \\ E-11510 Puerto Real, Cádiz, Spain
}

\begin{abstract}
Electrophoretic examination of 8 allozyme loci in 10 Spanish natural populations of Crassostrea angulata revealed a high level of genetic variability in terms of average allele number, polymorphism and mean heterozygosity. Significant departures of genotypic frequencies from the HardyWeinberg conditions, which could not be explained after pooling alleles, were displayed at some loci. All populations showed a deficit of heterozygotes, according to Selander's $D$ [(Het.observed Het expected)/Het expected; moreover, the mean $D$ index across populations was negative for the Lap, Mdh-1, Mdh-2, Me-2 and Xdh genes, consistent with $F_{\mathrm{TT}}$ estimates, which indicates deviations from Hardy-Weinberg equilibrium within the total population. In contrast, loci Me-1 and Pgi were at equilibrium and Pgm showed a heterozygous excess. Null alleles appeared in Lap and Mdh-1 loci, which could have been responsible for the disequilibrium, at least in Mdh-1, because the increase of frequencies of null alleles was also related to the increase in the deficit of heterozygotes. Although a high level of gene flow between populations was detected, heterogeneity in the allelic frequencies of Lap, $M d h-1, M d h-2, M e-2, P g m$ and $X d h$ genes was shown to occur. Mean $F_{S T}$ (F-statistic indicating deviations from Hardy-Weinberg equilibrium among populations) across loci was 0.031. meaning a slight genetic differentiation; however, no relationships were shown to exist between genetic distances, geographic distances and their location (estuarine or marine sites).
\end{abstract}

KEY WORDS: Crassostrea angulata Polymorphism Oysters Genetıc differentiation Allozymes

\section{INTRODUCTION}

Crassostrea angulata, known as Portuguese oyster, is an oyster species naturally located on the coast of the the southwest Iberian Peninsula, mainly at river mouths and heads of estuaries with suitable hard substrates (Marteil 1960). Adult oysters are sessile, with a tendency toward protandrous hermaphroditism: firsttime spawners are predominantly males, while older age classes often change to females. Simultaneous production of eggs and sperm by an individual is rare, and hence self-fertilization is minimal. Spawning takes place several times during the summer months, with both sexes synchronously releasing gametes in to the water column. Sperm and eggs remain viable for up to

\footnotetext{
- Addressee for correspondence.

E-mall: laureana.rebordinos@uca.es
}

5 and 24 h, respectively. Fertilization is external, and the zygote reaches a free-swimming planktonic larval stage within $48 \mathrm{~h}$, then the veliger larvae may swim and drift for 2 to $3 \mathrm{wk}$ and provide the potential for high dispersal (Korringa 1952)

There are contradictory data about the systematics of some Crassostrea species. C. sikamea had been considered a local variant of the Pacific oyster $C$. gigas because they occur sympatrically, although 2 types of C. sikamea oysters are known. Clear taxonomic separation between $C$. sikamea and $C$. gigas had not been established until recently, based on the concordance of results from gametic incompatibility, fixed alternate mitochondrial haplotypes and nuclear-encoded allozyme-frequency differences (Banks et al. 1993). Moreover, C. virginica and C. rhizophorae are considered to be closely related species or different varieties of the same species and they have the highest genetic similarity within the genus Crassostrea (Buroker et al. 
1979). Another species, C. rivularis, was thought to have 'red' and 'white' forms until they were recognized to be morphologically and biochemically distinct species, $C$. rivularis and $C$. iredalei, respectively (Gaffney \& Standish 1993). On the other hand, $C$. angulata and $C$. gigas have been classified as distinct species based on their geographical location, their fossil records and to some extent their morphology; however they cannot be distinguished in terms of larval morphology and karyotype (Thiriot-Quiévreux 1984).

There is an obvious interest in clari. fying the taxonomy of the genus in terms of interspecific hybridization that might yield small changes in commercial traits of the species, because these traits can be refined by selection and backcrossing. An important feature of this strategy for aquaculture is the rapid generation of novel genotypes in contrast to the gradual progress of conventional breeding. Nevertheless, according to Gaffney \& Standish (1993) there has not been unequivocal evidence for the formation of viable interspecific hybrids among species of the genus Crassostrea.

Published genetical data on Crassostrea angulata are scarce and incomplete and natural populations of the species have not been studied before. These populations were overexhausted from the 1940s until the 1970 s and nowadays they are not only of obvious taxonomic and ecological interest but also of economic importance, because they are sources of progenitors for hatcheries

Population genetics of marine molluscs is characterized by (1) significant and persistent departures of genotypic frequencies from the Hardy-Weinberg expectations (Foltz 1986b, Hare et al. 1996), (2) relationships between genetic structures and environmental changes (Koehn et al. 1980) and (3) the relationship between heterozygosities and/or allozyme genotypes and quantitative traits such as viabilities, growth rate, etc. (Zouros et al. 1988).

The aim of this work was to study the genetic variability, genetical structure and relationships of natural populations of Crassostrea angulata by analyzing allozyme variation in order to gain insight into the genetics and population structure of the species.

\section{MATERIAL AND METHODS}

Samples. Samples of Crassostrea angulata were taken from natural populations at 5 estuarine and 5 nearshore marine sites along approximately $200 \mathrm{~km}$ of the southwestern Spanish coast from the mouth of the Guadalquivir River to the Straits of Gibraltar (Fig. 1). The estuarine sites were Sanlúcar de Barrameda (SBD), located at the mouth of Guadalquivir River, and the nearby located Punta de Montijo (SBPM), Chipiona Faro (CHF) and Chipiona Corral (CCP). Barbate (BR) at the mouth of Barbate River was also an estuarine site, but far away from the others. The marine locations were: La Caleta (CLC), Santa María del Mar (CSMM), San Fernando (SFT), Sancti Petri (CTSP) and Vejer (VP)

Electrophoretic methods. Specimens were brought to the laboratory and, after being cleaned of fouling organisms, kept alive in a tank containing chlorated 
water and allowed to purge themselves for $24 \mathrm{~h}$. Starch gel electrophoresis was conducted using methods outlined by Selander et al. (1971). Six enzymatic systems controlled by 8 loci were studied: malic enzyme (E.C. 1.1.1.40) was controlled by the 2 loci $M e-1$ and $M e-2$; malate dehydrogenase (E.C. 1.1.1.37) by $M d h-1$ and Mdh-2; phosphoglucomutase (E.C. 5.4.2.2) by Pgm; phosphoglucoisomerase (E.C. 5.3.1.9) by Pgi leucine aminopeptidase (E.C. 3.4.11.1) by Lap; and xanthine dehydrogenase (E.C. 1.1.1.204) by Xdh. Activity zones were numbered from slower to faster migration, and within a locus alleles were assigned by numbers indicating their mobility relative to that of most common allele which was designated as 100 . Null alleles were referred to by the number ' 00 (Foltz 1986b).

Data analysis. Genetic variability was determined by using 3 estimators: (1) the unbiased estimate of heterozygosity for each locus $(h)$ and the average heterozygosity over loci $(H)$; (2) the percentage of polymorphic loci at the $95 \%$ and $99 \%$ levels $\left(P_{95}\right.$ and $P_{99}$ : the frequency of any allele of a particular gene does not exceed the the 0.95 and 0.99 values, respectively) and under non-criterion $\left(P_{\mathrm{nc}}\right.$ : every allele was considered without restriction of allelic frequencies); and (3) the average number of alleles per locus $\left(n_{d}\right)$ and the average number of alleles with frequencies $>0.05$ per locus $\left(n_{a p}\right)$. Average unbiased heterozygosity was calculated based on the methods of Nei (1978) and calculation of gene frequencies, conformance to HardyWeinberg equilibrium, $F_{\mathrm{ST}}$ determinations (indicating the deviance from Hardy-Weinberg equilibrium among populations) and unbiased genetic distance measures were performed with the BIOSYS program of Swofford \& Selander (1981). The degree of heterozygote deficiency below Hardy-Weinberg expectations was recorded as the $D$ index [(Het.observed Het.expected/Het -expected, where Het. is the number of heterozygotes].

To study the amount of gene flow between populations, the genetically effective number of migrants between populations, $N m$, was estimated using Wright's (1943) island model of population structure. Under this model genetic divergence is related to gene flow by the formula $N m=\left(1 / F_{\mathrm{ST}}-1\right) / 4$ using the average $F_{\text {ST }}$ across loci.

The genetic divergence between population samples based on Hedrick's (1971) distances were displayed by UPGMA (unweighted pair-group method using arithmetric averages) dendrograms (Sneath \& Sokal 1973).

\section{RESULTS}

The 8 genes studied were polymorphic, with allelic frequencies as shown in Table 1 Polymorphism ranged between 62.5 and $75 \%$ at the $P_{95}$ level however, it increased to $87 \%$ in 4 populations and to $100 \%$ in 3 populations at the $P_{99}$ level and under noncriterion one more population than this reached $100 \%$. The average heterozygosity $(H)$ in populations ranged from a maximum value of $0.382 \pm 0.086$ in CCP, which also had the highest average allele number $\left(n_{\mathrm{a}}=3.38 \pm\right.$ 0.56 ), to a minimal value of $0.292 \pm 0.103$ in $\mathrm{BR}$, which had $n_{\mathrm{c}}=2.63 \pm 0.53$. SBPM showed the lowest $n_{\mathrm{c}}$ of $2.50 \pm 0.57$ with an $H$ of $0.297 \pm 0.016$. When only alleles present at frequencies larger than 0.05 were considered, their numbers, $n_{\text {dp }}$, varied from $2.00 \pm 0.33$ in BR to $2.63 \pm 0.50$ in CCP and $2.63 \pm 0.46$ in $\mathrm{CHF}$

Pgi, Pgm, Me- 1 and Mdh- 2 genes appeared to be at equilibrium in all populations except $M d h-2$ in CCP and Pgm in CSMM However, Mdh-1, Me-2, Xdh and Lap reported significant $\chi^{2}$ fit to Hardy-Weinberg conditions (Table 2) When pooling of alleles was carried out, nonsignificant values of $\chi^{2}$ were obtained for the Lap gene in most populations, indicating a conformance to Hardy-Weinberg proportion expectations. However, the $\chi^{2}$ values for $M e-2$ in SBD and $M d h-1$ in CHF and CLC decreased and did not change for the $X d h$ gene. According to results for the $D$ index, genes can be put into 2 different categories: (1) negative values indicating high heterozygote deficiencies, as for Xdh (-0.562), Mdh-1 (-0.403), Me-2 (-0.268), Lap $(-0.247)$ and $M d h-2(-0.150)$; and (2) close to zero or positive values, as for Me-1 (-0.027), Pgi (0.085) and Pgm (0.230). Mean $D$ within populations reported negative values for all of them. Coincident results were displayed by $F$-statistics so that $F_{\text {IT }}$ (indicating the deviance from Hardy-Weinberg equilibrium within the total population) displayed positive values at most of the loci, indicating a general deficiency of heterozygotes. Of those not showing positive results, $\mathrm{Pgm}$ produced a large negative value $(-0.204)$ but $\mathrm{Me}-1$ $(-0.018)$ and Pgi $(-0.069)$ were close to zero. F $F_{\text {IS }}$ contributed the most to $F_{\mathrm{IT}}$ in all values, indicating deviations from Hardy-Weinberg proportions within populations (Table 2). The largest geographic differentiation in allelic frequencies was displayed by $M d h-1$ $\left(F_{\mathrm{ST}}=0.078\right)$ and $X d h\left(F_{\mathrm{ST}}=0.052\right) \cdot \chi^{2}$ values of contingency indicated that Lap,Mdh-1, Me-2,Pgm and Xdh were statistically significant at $\mathrm{p}<0.001, M d h-2$ was significant at $\mathrm{p}<0.05$ and Em- 1 and Pgi were homogenous. The average $N_{m}$ was $7.81 \pm 3.28$ ranging from 17.61 in $\mathrm{Me}-1$ to 2.96 in $\mathrm{Mdh}-1$

The maximum unbiased genetic distance of Nei (1978) was 0.038 between CLC and CHF and the minimum was 0.000 between SBPM and SBD. According to Hedrick's (1971) data, the maximum was 0.074 between SBD and CTSP and the minimum 0.010 between CCP and CHF (Table 3) The genetical differentiation between populations can be seen more 
Table 1. Crassostrea angulata. Genetıc variability at 8 loci in 10 natural populations. $h$ : heterozygosity; $N$ : sample size: $P_{95}, P_{99:}$ $p_{\text {c: }}$ percent of loci polymorphic at $95 \%, 99 \%$ and non-criterion levels, respectively; $H$ : average heterozygosity; $n_{\alpha}$ : average number of alleles per locus; $n_{\mathrm{ap}}$ : average number of alleles with frequencies $>0.5$ per locus. SE: standard error. See Fig. 1 for locality abbreviations

\begin{tabular}{|c|c|c|c|c|c|c|c|c|c|c|c|}
\hline Locus & Allele & SBD & SBPM & $\mathrm{CHF}$ & $\mathrm{CCP}$ & CLC & CSMM & SFT & CTSP & $V P$ & $B R$ \\
\hline \multirow[t]{7}{*}{ Lap } & $(\mathrm{N})$ & (38) & $(51)$ & (56) & $(50)$ & (63) & $(47)$ & (51) & (57) & (45) & (48) \\
\hline & .73 & 0.000 & 0.000 & 0.000 & 0.000 & 0.000 & 0.043 & 0.000 & 0.000 & 0.022 & 0.000 \\
\hline & $\cdot 91$ & 0.132 & 0.127 & 0.143 & 0.100 & 0.190 & 0.170 & 0.216 & 0.123 & 0.133 & 0.094 \\
\hline & $\cdot 100$ & 0.776 & 0.873 & 0.652 & 0.670 & 0.714 & 0.638 & 0.716 & 0.711 & 0.778 & 0.740 \\
\hline & $\cdot 122$ & 0.092 & 0.000 & 0.116 & 0.110 & 0.063 & 0.149 & 0.059 & 0.167 & 0.067 & 0.167 \\
\hline & $\cdot 00$ & 0.000 & 0.000 & 0.089 & 0.120 & 0.032 & 0.000 & 0.000 & 0.000 & 0.000 & 0.000 \\
\hline & $h$ & 0.376 & 0.225 & 0.538 & 0.520 & 0.952 & 0.545 & 0.442 & 0.456 & 0.377 & 0.421 \\
\hline \multirow[t]{5}{*}{$M d h-1$} & (N) & $(60)$ & $(46)$ & $(36)$ & $(80)$ & (71) & (43) & (55) & (59) & (42) & $(50)$ \\
\hline & $\cdot 85$ & 0.017 & 0.011 & 0.056 & 0.038 & 0.014 & 0.012 & 0.018 & 0.034 & 0.024 & 0.010 \\
\hline & $\cdot 100$ & 0.917 & 0.989 & 0.889 & 0.738 & 0.972 & 0.826 & 0.982 & 0.966 & 0.929 & 0.990 \\
\hline & $\cdot 00$ & 0.067 & 0.000 & 0.056 & 0.225 & 0.014 & 0.163 & 0.000 & 0.000 & 0.048 & 0.000 \\
\hline & $h$ & 0.156 & 0.022 & 0.207 & 0.407 & 0.056 & 0.295 & 0.036 & 0.066 & 0.137 & 0.020 \\
\hline \multirow[t]{4}{*}{$M d h-2$} & $(\mathrm{~N})$ & $(60)$ & $(47)$ & (39) & $(79)$ & (71) & (58) & (55) & (59) & (49) & $(50)$ \\
\hline & $\cdot 100$ & 1.000 & 1.000 & 0.962 & 0.981 & 1.000 & 1.000 & 1.000 & 0.992 & 0.990 & 1.000 \\
\hline & $\cdot 118$ & 0.000 & 0.000 & 0.038 & 0.019 & 0.000 & 0.000 & 0.000 & 0.008 & 0.010 & 0.000 \\
\hline & h & 0.000 & 0.000 & 0.075 & 0.037 & 0.000 & 0.000 & 0.000 & 0.017 & 0.020 & 0.000 \\
\hline \multirow[t]{5}{*}{ Me-1 } & $(\mathrm{N})$ & $(60)$ & (47) & (43) & $(58)$ & (71) & (45) & (65) & (62) & (52) & $(50)$ \\
\hline & .99 & 0.025 & 0.000 & 0.023 & 0.043 & 0.007 & 0.011 & 0.015 & 0.008 & 0.048 & 0.000 \\
\hline & $\cdot 100$ & 0.975 & 1.000 & 0.977 & 0.957 & 0.986 & 0.989 & 0.985 & 0.992 & 0.952 & 1.000 \\
\hline & $\cdot 101$ & 0.000 & 0.000 & 0.000 & 0.000 & 0.007 & 0.000 & 0.000 & 0.000 & 0.000 & 0.000 \\
\hline & $h$ & 0.049 & 0.000 & 0.046 & 0.083 & 0.028 & 0.022 & 0.031 & 0.016 & 0.092 & 0.000 \\
\hline \multirow[t]{5}{*}{$\mathrm{Me}-2$} & $(\mathrm{~N})$ & $(58)$ & $(46)$ & (92) & $(86)$ & (59) & (56) & (51) & $(60)$ & (51) & $(47)$ \\
\hline & .90 & 0.207 & 0.207 & 0.092 & 0.128 & 0.068 & 0.134 & 0.118 & 0.108 & 0.108 & 0.032 \\
\hline & $\cdot 100$ & 0.586 & 0.609 & 0.772 & 0.756 & 0.551 & 0.670 & 0.588 & 0.650 & 0.647 & 0.660 \\
\hline & $\cdot 110$ & 0.207 & 0.185 & 0.136 & 0.116 & 0.381 & 0.196 & 0.294 & 0.242 & 0.245 & 0.309 \\
\hline & $h$ & 0.576 & 0.559 & 0.379 & 0.401 & 0.551 & 0.500 & 0.559 & 0.512 & 0.515 & 0.474 \\
\hline \multirow[t]{5}{*}{ Pgi } & $(\mathrm{N})$ & (66) & (47) & (76) & (61) & (68) & (55) & (55) & (58) & $(46)$ & $(50)$ \\
\hline & $\cdot 77$ & 0.098 & 0.117 & 0.132 & 0.164 & 0.059 & 0.118 & 0.082 & 0.086 & 0.043 & 0.060 \\
\hline & $\cdot 100$ & 0.902 & 0.883 & 0.855 & 0.828 & 0.941 & 0.882 & 0.918 & 0.914 & 0.957 & 0.940 \\
\hline & $\cdot 157$ & 0.000 & 0.000 & 0.013 & 0.008 & 0.000 & 0.000 & 0.000 & 0.000 & 0.000 & 0.000 \\
\hline & $h$ & 0.179 & 0.209 & 0.253 & 0.290 & 0.112 & 0.210 & 0.152 & 0.159 & 0.084 & 0.114 \\
\hline \multirow[t]{10}{*}{$P_{g m}$} & $(\mathrm{~N})$ & $(47)$ & $(44)$ & (55) & $(47)$ & $(65)$ & (54) & $(50)$ & (61) & $(42)$ & $(45)$ \\
\hline & .80 & 0.011 & 0.000 & 0.000 & 0.000 & 0.000 & 0.000 & 0.000 & 0.000 & 0.000 & 0.000 \\
\hline & $\cdot 90$ & 0.043 & 0.000 & 0.000 & 0.021 & 0.054 & 0.019 & 0.010 & 0.000 & 0.048 & 0.011 \\
\hline & $\cdot 94$ & 0.096 & 0.023 & 0.055 & 0.053 & 0.038 & 0.056 & 0.060 & 0.057 & 0.060 & 0.044 \\
\hline & $\cdot 96$ & 0.021 & 0.057 & 0.236 & 0.074 & 0.230 & 0.046 & 0.060 & 0.131 & 0.107 & 0.011 \\
\hline & $\cdot 100$ & 0.309 & 0.341 & 0.282 & 0.319 & 0.315 & 0.324 & 0.360 & 0.230 & 0.214 & 0.267 \\
\hline & $\cdot 114$ & 0.213 & 0.170 & 0.209 & 0.181 & 0.277 & 0.130 & 0.140 & 0.189 & 0.226 & 0.311 \\
\hline & $\cdot 132$ & 0.298 & 0.386 & 0.218 & 0.319 & 0.262 & 0.417 & 0.370 & 0.369 & 0.345 & 0.356 \\
\hline & $\cdot 138$ & 0.011 & 0.023 & 0.000 & 0.032 & 0.031 & 0.000 & 0.000 & 0.025 & 0.000 & 0.000 \\
\hline & $h$ & 0.767 & 0.709 & 0.777 & 0.762 & 0.755 & 0.705 & 0.714 & 0.761 & 0.776 & 0.711 \\
\hline \multirow[t]{5}{*}{$X d h$} & $(\mathrm{~N})$ & (48) & (37) & $(80)$ & (42) & (33) & (43) & $\{50\}$ & $(48)$ & (39) & $(40)$ \\
\hline & .99 & 0.240 & 0.243 & 0.131 & 0.131 & 0.242 & 0.302 & 0.240 & 0.438 & 0.167 & 0.100 \\
\hline & $\cdot 100$ & 0.385 & 0.459 & 0.706 & 0.607 & 0.318 & 0.384 & 0.480 & 0.479 & 0.551 & 0.438 \\
\hline & $\cdot 101$ & 0.375 & 0.297 & 0.162 & 0.262 & 0.439 & 0.314 & 0.480 & 0.083 & 0.282 & 0.463 \\
\hline & $h$ & 0.660 & 0.650 & 0.460 & 0.552 & 0.657 & 0.671 & 0.640 & 0.578 & 0.596 & 0.592 \\
\hline$P_{95}$ & & 75.0 & 62.5 & 75.0 & 75.0 & 62.5 & 75.0 & 62.5 & 62.5 & 62.5 & 62.5 \\
\hline$P_{99}$ & & 87.5 & 62.5 & 100.0 & 100.0 & 87.5 & 87.5 & 87.5 & 75.0 & 100.0 & 75.0 \\
\hline$P_{\mathrm{nc}}$ & & 87.5 & 75.0 & 100.0 & 100.0 & 87.5 & 87.5 & 87.5 & 100.0 & 100.0 & 75.0 \\
\hline $\begin{array}{l}H \\
(\mathrm{SE})\end{array}$ & & $\begin{array}{c}0.346 \\
(0.103)\end{array}$ & $\begin{array}{c}0.297 \\
(0.016)\end{array}$ & $\begin{array}{c}0.342 \\
(0.088)\end{array}$ & $\begin{array}{c}0.382 \\
(0.086)\end{array}$ & $\begin{array}{c}0.326 \\
(0.110)\end{array}$ & $\begin{array}{c}0.369 \\
(0.098)\end{array}$ & $\begin{array}{c}0.322 \\
(0.106)\end{array}$ & $\begin{array}{c}0.321 \\
(0.103)\end{array}$ & $\begin{array}{c}0.325 \\
(0.100)\end{array}$ & $\begin{array}{c}0.292 \\
(0.103)\end{array}$ \\
\hline $\begin{array}{l}n_{a} \\
\text { (SE) }\end{array}$ & & $\begin{array}{c}3.13 \\
(0.74)\end{array}$ & $\begin{array}{c}2.50 \\
(0.57)\end{array}$ & $\begin{array}{l}3.13 \\
(0.35)\end{array}$ & $\begin{array}{c}3.38 \\
(0.56)\end{array}$ & $\begin{array}{c}3.25 \\
(0.62)\end{array}$ & $\begin{array}{c}3.13 \\
(0.64)\end{array}$ & $\begin{array}{c}2.88 \\
(0.55)\end{array}$ & $\begin{array}{c}2.88 \\
(0.48)\end{array}$ & $\begin{array}{c}3.13 \\
(0.48)\end{array}$ & $\begin{array}{c}2.63 \\
(0.53)\end{array}$ \\
\hline $\begin{array}{l}n_{\mathrm{ap}} \\
\text { (SE) }\end{array}$ & & $\begin{array}{c}2.38 \\
(0.37)\end{array}$ & $\begin{array}{c}2.13 \\
(0.40\}\end{array}$ & $\begin{array}{c}2.63 \\
(0.46)\end{array}$ & $\begin{array}{c}2.63 \\
(0.50)\end{array}$ & $\begin{array}{c}2.38 \\
(0.50)\end{array}$ & $\begin{array}{c}2.38 \\
(0.37)\end{array}$ & $\begin{array}{c}2.38 \\
(0.50)\end{array}$ & $\begin{array}{c}2.38 \\
(0.50)\end{array}$ & $\begin{array}{c}2.25 \\
(0.53)\end{array}$ & $\begin{array}{c}2.00 \\
(0.33)\end{array}$ \\
\hline
\end{tabular}


Table 2. Crassostrea angulata. Chi-squared values of fit to genotype Hardy-Weinberg proportions both without $\left(\chi^{2}\right)$ and with $\left(\chi^{2} p\right)$ pooling of alleles in 10 populations. $D$ : heterozygote deficiency, $\chi_{c}^{2}$ : chi-squared value of contigency tests of allelic frequencies, Nm: gene flow value. F-statistics (Nei 1977) indicate deviations from Hardy-Weinberg proportions within the total population $\left(F_{T T}\right)$, within populations $\left(F_{I S}\right)$, among populations $\left(F_{S T}\right)$. -: monomorphic locus. See Fig. 1 for locality abbreviations. na: non applicable. SE: standard error; ${ }^{*} p<0.05 ; \cdots p<0.01 ; \cdots p<0.001$

\begin{tabular}{|c|c|c|c|c|c|c|c|c|c|c|}
\hline \multicolumn{2}{|c|}{ Population } & \multirow{2}{*}{$\begin{array}{l}\text { Lap } \\
0.29 \\
0.00 \\
0.079\end{array}$} & \multirow{2}{*}{$\begin{array}{l}M d h-1 \\
45.76 \cdots \\
27.85 \cdots \\
-0.785\end{array}$} & \multirow{2}{*}{$\begin{array}{c}\text { Mdh-2 } \\
- \\
\text { na }\end{array}$} & \multirow{2}{*}{$\begin{array}{l}\text { Me-1 } \\
0.00 \\
0.84 \\
0.026\end{array}$} & \multirow{2}{*}{$\begin{array}{c}M e-2 \\
6.34 \\
\text { na } \\
-0.215\end{array}$} & \multirow{2}{*}{$\begin{array}{l}\text { Pgi } \\
0.08 \\
7.79 \cdots \\
0.109\end{array}$} & \multirow{2}{*}{$\begin{array}{l}\text { Pgm } \\
25.66 \\
11.19 \cdots \\
0.317\end{array}$} & \multirow{2}{*}{$\begin{array}{c}x d b \\
24.62 \cdots \\
0.317\end{array}$} & \multirow{2}{*}{$\begin{array}{c}\text { Mean } D(\mathrm{SE}) \\
-0.044(0.144)\end{array}$} \\
\hline SBD & $\begin{array}{l}\chi^{2} \\
\chi_{p}^{2} \\
D\end{array}$ & & & & & & & & & \\
\hline SBPM & $\begin{array}{l}\chi^{2} \\
\chi^{2} p \\
D\end{array}$ & $\begin{array}{l}0.25 \\
\text { na } \\
0.146\end{array}$ & $\begin{array}{l}0.00 \\
\text { na } \\
0.011\end{array}$ & - & - & $\begin{array}{c}6.52 \\
4.91 \\
-0.331\end{array}$ & $\begin{array}{l}0.10 \\
\text { na } \\
0.133\end{array}$ & $\begin{array}{c}23.27 \\
15.48 \cdots \\
0.426\end{array}$ & $\begin{array}{l}20.01 \cdots \\
10.16 \cdots \\
-0.536\end{array}$ & $-0.025(0.143)$ \\
\hline $\mathrm{CHF}$ & $\begin{array}{l}\chi^{2} \\
\chi^{2} p \\
D\end{array}$ & $\begin{array}{c}46.12 \cdots \\
0.24 \\
-0.196\end{array}$ & $\begin{array}{c}20.03 \cdots \\
3.51 \\
-0.455\end{array}$ & $\begin{array}{l}0.00 \\
\text { na } \\
0.040\end{array}$ & $\begin{array}{l}0 \\
\text { na } \\
0.024\end{array}$ & $\begin{array}{l}33.74 \cdots \\
27.10 \cdots \\
-0.510\end{array}$ & $\begin{array}{l}0.863 \\
1.15 \\
0.153\end{array}$ & $\begin{array}{c}2.82 \\
0.03 \\
-0.009\end{array}$ & $\begin{array}{c}108.00 \cdots \\
54.52 \cdots \\
-0.863\end{array}$ & $-0.227(0.124)$ \\
\hline $\mathrm{CCP}$ & $\begin{array}{l}\chi^{2} \\
\chi_{p}^{2} \\
D\end{array}$ & $\begin{array}{c}42.50 \cdots \\
7.11 \cdots \\
-0.417\end{array}$ & $\begin{array}{l}74.33 \cdots \\
48.71 \cdots \\
-0.814\end{array}$ & $\begin{array}{c}8.51 \cdots \\
\text { na } \\
-0.660\end{array}$ & $\begin{array}{c}0.00 \\
\text { na } \\
0.045\end{array}$ & $\begin{array}{l}56.07 \cdots \\
30.58 \cdots \\
-0.621\end{array}$ & $\begin{array}{c}0.00 \\
0.00 \\
-0.031\end{array}$ & $\begin{array}{l}27.49 \\
0.31 \\
0.073\end{array}$ & $\begin{array}{l}57.20 \cdots \\
34.96 \cdots \\
-0.913\end{array}$ & $-0.417(0.141)$ \\
\hline CLC & $\begin{array}{l}\chi^{2} \\
\chi^{2} p \\
D\end{array}$ & $\begin{array}{c}38.05^{\cdots} \\
4.57^{\circ} \\
-0.328\end{array}$ & $\begin{array}{c}17.84^{\cdots} \\
3.99^{\circ} \\
-0.489\end{array}$ & - & $\begin{array}{l}0.00 \\
0.00 \\
0.011\end{array}$ & $\begin{array}{c}4.75 \\
2.06 \\
-0.256\end{array}$ & $\begin{array}{c}0.00 \\
\text { na } \\
0.062\end{array}$ & $\begin{array}{c}17.99 \\
5.48 \\
0.190\end{array}$ & $\begin{array}{l}0.068 \\
0.040 \\
0.063\end{array}$ & $-0.125(0.091)$ \\
\hline CSMM & $\begin{array}{l}\chi^{2} \\
\chi^{2} p \\
D\end{array}$ & $\begin{array}{c}51.77 \cdots \\
2.44 \\
-0.369\end{array}$ & $\begin{array}{l}36.72 \cdots \\
31.09 \cdots \\
-0.920\end{array}$ & - & $\begin{array}{l}0.00 \\
\text { na } \\
0.011\end{array}$ & $\begin{array}{c}15.27 \cdots \\
7.39 \cdots \\
-0.351\end{array}$ & $\begin{array}{l}0.189 \\
\text { na } \\
0.134\end{array}$ & $\begin{array}{c}33.97^{\circ} \\
25.38^{\cdots} \cdots \\
0.431\end{array}$ & $\begin{array}{c}21.43^{\cdots} \\
16.44 \cdots \\
-0.544\end{array}$ & $-0.230(0.172)$ \\
\hline SFT & $\begin{array}{l}\chi^{2} \\
\chi^{2} p \\
D\end{array}$ & $\begin{array}{c}7.17 \\
2.92 \\
-0.328\end{array}$ & $\begin{array}{l}0.00 \\
\text { na } \\
0.019\end{array}$ & - & $\begin{array}{l}0.00 \\
\mathrm{na} \\
0.616\end{array}$ & $\begin{array}{r}10.29^{*} \\
5.28^{*} \\
0.363\end{array}$ & $\begin{array}{c}0.00 \\
\text { na } \\
0.089\end{array}$ & $\begin{array}{c}23.66 \\
15.37 \cdots \\
0.415\end{array}$ & $\begin{array}{l}36.02 \cdots \\
21.09 \cdots \\
-0.653\end{array}$ & $-0.029(0.170)$ \\
\hline CTSP & $\begin{array}{l}\chi^{2} \\
\chi_{p}^{2} \\
D\end{array}$ & $\begin{array}{c}22.97 \cdots \\
1.39 \\
-0.263\end{array}$ & $\begin{array}{l}0.00 \\
\text { na } \\
0.035\end{array}$ & $\begin{array}{l}0.00 \\
\text { na } \\
0.009\end{array}$ & $\begin{array}{l}0.00 \\
\mathrm{na} \\
0.008\end{array}$ & $\begin{array}{c}38.02 \cdots \\
40.86 \cdots \\
0.639\end{array}$ & $\begin{array}{l}0.014 \\
\mathrm{na} \\
0.094\end{array}$ & $\begin{array}{l}4.30 \\
2.58 \\
0.043\end{array}$ & $\begin{array}{l}75.53 \cdots \\
37.62 \cdots \\
-0.927\end{array}$ & $-0.045(0.154)$ \\
\hline$\vee P$ & $\begin{array}{l}\chi^{2} \\
\chi_{p}^{2} \\
D\end{array}$ & $\begin{array}{c}12.30 \\
1.40 \\
-0.284\end{array}$ & $\begin{array}{c}23.55 \cdots \\
9.45^{\cdots} \\
-0.64\end{array}$ & $\begin{array}{c}0.00 \\
\text { na } \\
0.010\end{array}$ & $\begin{array}{l}0.00 \\
\mathrm{na} \\
0.051\end{array}$ & $\begin{array}{c}18.28 \cdots \\
6.83 \cdots \\
-0.401\end{array}$ & $\begin{array}{l}0.00 \\
\text { na } \\
0.045\end{array}$ & $\begin{array}{c}17.60 \\
6.11 \\
0.180\end{array}$ & $\begin{array}{l}33.90^{\cdots} \\
19.22 \cdots \\
-0.739\end{array}$ & $-0.222(0.122)$ \\
\hline BR & $\begin{array}{l}\chi^{2} \\
\chi_{p}^{2} \\
D\end{array}$ & $\begin{array}{c}18.94 \cdots \\
3.09 \\
-0.350\end{array}$ & $\begin{array}{l}0.00 \\
\text { na } \\
0.010\end{array}$ & - & $\begin{array}{l}16.77 \cdots \\
11.27 \cdots \\
-0.546\end{array}$ & - & $\begin{array}{l}0.00 \\
\text { na } \\
0.064\end{array}$ & $\begin{array}{l}8.93 \\
2.25 \\
0.232\end{array}$ & $\begin{array}{c}31.23 \cdots \\
20.22 \cdots \\
-0.701\end{array}$ & $-0.215(0.152)$ \\
\hline $\begin{array}{c}\text { Mean } D \\
(\mathrm{SE})\end{array}$ & & $\begin{array}{c}-0.247 \\
(0.053)\end{array}$ & $\begin{array}{c}-0.403 \\
(0.123)\end{array}$ & $\begin{array}{c}-0.150 \\
(0.170)\end{array}$ & $\begin{array}{r}-0.027 \\
\{0.097\}\end{array}$ & $\begin{array}{c}-0.268 \\
(0.121)\end{array}$ & $\begin{array}{c}0.085 \\
(0.017)\end{array}$ & $\begin{array}{c}0.230 \\
(0.052)\end{array}$ & $\begin{array}{c}0.562 \\
(0.126)\end{array}$ & \\
\hline $\begin{array}{l}F_{\mathrm{IT}} \\
F_{\mathrm{IS}} \\
F_{\mathrm{ST}}\end{array}$ & & $\begin{array}{l}0.289 \\
0.273 \\
0.023\end{array}$ & $\begin{array}{l}0.691 \\
0.665 \\
0.078\end{array}$ & $\begin{array}{l}0.160 \\
0.144 \\
0.019\end{array}$ & $\begin{array}{r}-0.018 \\
-0.032 \\
0.014\end{array}$ & $\begin{array}{l}0.432 \\
0.417 \\
0.026\end{array}$ & $\begin{array}{r}-0.069 \\
-0.086 \\
0.016\end{array}$ & $\begin{array}{r}-0.204 \\
-0.225 \\
0.018\end{array}$ & $\begin{array}{l}0.652 \\
0.633 \\
0.052\end{array}$ & $\begin{array}{c}\text { Mean F-statistic } \\
0.258 \\
0.234 \\
0.031\end{array}$ \\
\hline$\chi_{c}^{2}$ & & $104.13 \cdots$ & $129.85 \cdots$ & $19.21^{\circ}$ & 22.13 & $69.59 \cdots$ & 27.16 & $135.97 \cdots$ & $105.56 \cdots$ & $\begin{array}{c}\text { Total } \chi^{2} c \\
613.61 \cdots\end{array}$ \\
\hline $\mathrm{Nm}$ & & 10.62 & 2.96 & 12.91 & 17.61 & 9.37 & 15.38 & 13.64 & 4.56 & $\begin{array}{c}\text { Mean Nm }( \pm \mathrm{SE}) \\
7.81 \pm 3.28\end{array}$ \\
\hline
\end{tabular}

clearly in Fig. 2, which also indicates no relationships between genetic distances and geographic ones and their location (estuarine or marine).

\section{DISCUSSION}

The 2 features that stand out in this analysis are the high level of genetic variability and the slight genetic differentiation between local, natural populations of Crassostrea angulata. The enzymes studied were chosen randomly and not due to their variability; in fact the 2 systems controlled by 4 of the analysed loci (Mdh-1, Mdh-2, Me-1 and $M e-2$ ) had been described as monomorphic in C. virginica (Foltz 1986b). Moreover, Got1 (glutamate oxaloacetate transaminase), Cat (catalase) and Sod (superoxide dismutase) also appeared variable (data not shown). Presence of a high 
Table 3. Crassostrea angulata.Genetic distances (Hedrick 1971) above diagonal and unbiased genetic distances (Nei 1978) below diagonal, based on 8 loci for 10 populations. See Fig. 1 for locality abbreviations

\begin{tabular}{|lcccccccccc|}
\hline Population & CCP & CHF & CTSP & VP & SFT & CSMM & SBD & SBPM & BR & CLC \\
\hline CCP & - & 0.010 & 0.040 & 0.037 & 0.041 & 0.029 & 0.031 & 0.037 & 0.056 & 0.064 \\
CHF & 0.006 & - & 0.043 & 0.032 & 0.035 & 0.026 & 0.020 & 0.035 & 0.047 & 0.055 \\
CTSP & 0.025 & 0.019 & - & 0.073 & 0.044 & 0.072 & 0.074 & 0.040 & 0.040 & 0.071 \\
VP & 0.010 & 0.009 & 0.009 & - & 0.035 & 0.041 & 0.027 & 0.042 & 0.057 & 0.048 \\
SFT & 0.019 & 0.018 & 0.008 & 0.001 & - & 0.041 & 0.038 & 0.026 & 0.033 & 0.056 \\
CSMM & 0.008 & 0.022 & 0.010 & 0.008 & 0.004 & - & 0.023 & 0.045 & 0.049 & 0.043 \\
SBD & 0.016 & 0.024 & 0.013 & 0.003 & 0.002 & 0.003 & - & 0.046 & 0.054 & 0.038 \\
SBPM & 0.018 & 0.022 & 0.011 & 0.003 & 0.001 & 0.008 & 0.000 & - & 0.036 & 0.072 \\
BR & 0.023 & 0.027 & 0.023 & 0.004 & 0.008 & 0.013 & 0.005 & 0.011 & - & 0.055 \\
CLC & 0.034 & 0.038 & 0.022 & 0.009 & 0.005 & 0.012 & 0.003 & 0.011 & 0.003 & - \\
\hline
\end{tabular}

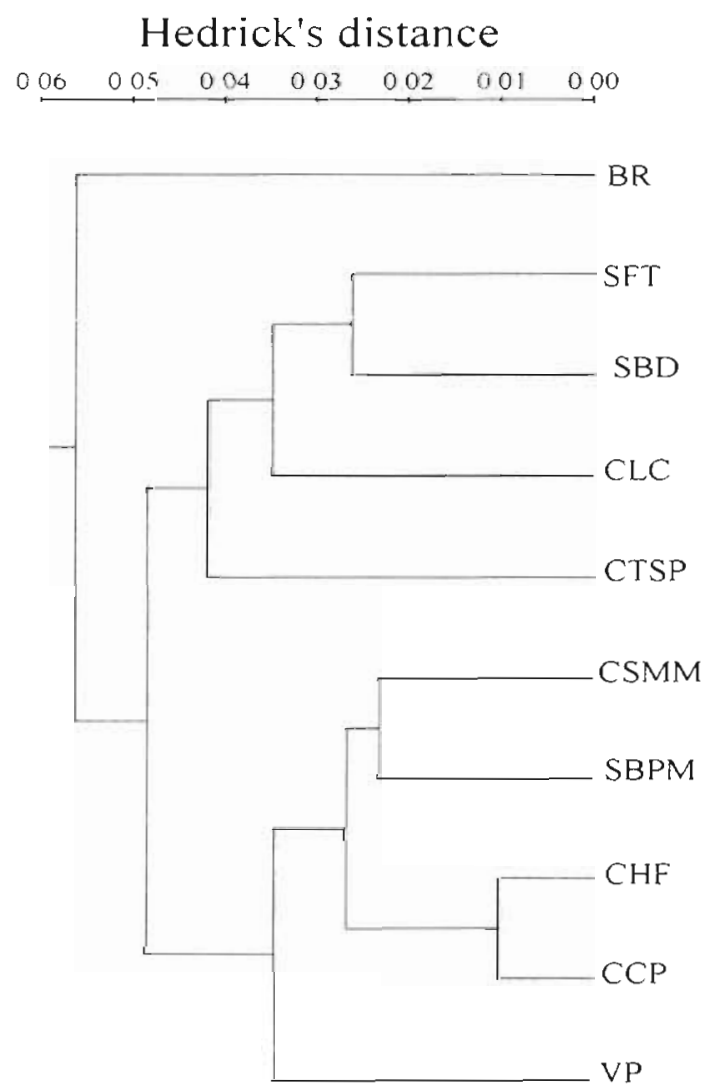

Fig. 2. Crassostrea angulata. UPGMA dendrogram showing relationshıps between populations based on Hedrick's distances in Table 3. See Fig. 1 for locality abbreviations

number of alleles at low frequencies has been described in bivalves (Sarver et al. 1992) and has to be considered in relation to the mean allele number $\left(n_{\mathrm{a}}\right)$ because the restricted distribution of alleles that occurred at frequencies less than 0.03 to 0.04 can be attributed to sampling.

The $D$ index showed a deficiency of heterozygotes in all populations, with the extent of homozygous excess varying greatly among loci, as has been repeatedly observed between marine molluscs (Singh \& Green 1984, Staton \& Felder 1995). One of the most extended theories reported for explaining this fact is the presence of null alleles in populations, because active/null heterozygotes can be misinterpreted to be active homozygotes. Null alleles have been reported in leucine aminopeptidase and mânuusephusplude isomerase in C. virginica (Foltz 1986a) and in leucine aminopeptidase in Mytilus edulis (Mallet et al. 1985). Experimental evidence in favour of null alleles as a possible cause of heterozygote deficiency in C. virginica has been obtained by Foltz (1986a).

A negative $D$ index value was found for the Lap gene in all populations except in SBPM (here both null alleles were missing and the $\chi^{2}$ of fit to Hardy-Weinberg conditions was not significant). SBD, VP and SFT populations exhibited a negative $D$ value, but their genotypic proportions were not statistically significant. Populations BR, CTSP and CSMM, with statistically significant $\chi^{2}$ values, showed nonsignificant $\chi^{2}$ values when pooling of alleles was carried out; CHF, CCP and CLC were the populations that showed null alleles. CHF also reported non-significant $\chi^{2}$ values on Lap and Mdh-1 genes after pooling; however, CLC and $\mathrm{CCP}$, in which null alleles appeared at frequencies of 0.032 and 0.120 , yielded $a \chi^{2}$ values of fit significant at $\mathrm{p}<0.05$ and $\mathrm{p}<0.01$ after pooling, respectively.

For the Mdh-1 locus, a relationship between departures from Hardy-Weinberg conditions and presence of null alleles occurred such that departures increased with increasing null allele frequency. Populations without null alleles were under Hardy-Weinberg equilibrium (SBPM, SFT, CTSP and BR); however populations displaying null alleles yielded both an $\chi^{2}$ of fit significant at $\mathrm{p}<0.001$ and negative $D$ values. After pooling of alleles, the $\chi^{2}$ value became nonsignificant for CHF and decreased to $p<0.01$ for VP and to $p<$ 0.05 for CLC; hence there is no clear evidence that null alleles are solely responsible for the heterozygote 
deficit, which could be due to both the presence of null alleles and alleles at low frequencies. However, in SBD with a null allele frequency of 0.067 , in CCP with 0.225 and in CSMM with 0.163 , the $\chi^{2}$ of fit after pooling remained significant, although all of them showed frequencies lower than 0.04 for the allele $M d h-1 \cdot 85$. At the same time, negative $D$ values increased with the frequency of null alleles, so that SBD with a null frequency of 0.067 had a $D$ value of -0.785 , CCP with 0.225 had $D=-0.814$ and CSMM with 0.295 had $D=$ -0.920 . This is in agreement with Siegesmund (1985), who reported that frequencies larger than 0.05 for null alleles could have a significant impact on the genetic structure of populations, including apparent deficiencies in the number of heterozygous individuals. However, except for species with duplicate genomes or loci (in which null alleles have been interpreted as being part of the process of gene silencing), the biological significance of allozyme null alleles is poorly understood (Rebordinos \& Pérez de la Vega 1987).

The other loci deviations can only be attributed to null alleles if it is assumed that null allele homozygote is lethal, since no non-staining individuals were observed, which would imply that the silent alleles occur in the heterozygote form only.

Another explanation for genotypic frequency departures could be the occurrence of selective forces causing post-settlement, micro-geographical differentiation, because if functional differences exist among genotypes and if certain heterozygotes represent disadvantageous levels of enzyme activity it could be assumed that natural selection will act against those heterozygous genotypes and thus account for the observed heterozygote deficiency. Although in most cases the action of selection at gene loci is difficult to demonstrate experimentally, evidence for the correlation of changes in allele frequencies with heterogeneous environmental parameters has been used to infer selection in natural populations of different species (Beaumont 1982). In this sense, higher frequencies of homozygotes have been found in juveniles than in adults, possibly as a result of selection against homozygosity (Koehn et al. 1976). A case of heterozygosis dependent growth has been established in the American oyster (Sing \& Zouros 1978), and studies in other bivalve species point to selection as a major factor causing heterozygote deficiency (Beaumont et al. 1983, Hilbish \& Koehn 1985).

Restricted gene flow between populations has been claimed to be responsible for the departure from equilibrium proportions. $\mathrm{Nm}$ indicates the relative strengths of gene flow and genetic drift, which will lead to substantial local differentiation if $N m<1$ but not if $N m>1$ (Slatkin 1987). $N m$ estimates from all loci (Table 2) are consistently above 1 and indicate that gene flow is sufficiently strong to prevent genetic drift from causing more local genetic differentiation. The locus Mdh-1 differs sufficiently from the others and itself or a locus closely linked could be subject to strong natural selection, favouring different alleles in different populations.

Mdh- 2 had a $D$ value of -0.150 , but was at HardyWeinberg equilibrium in all populations except CCP $(\mathrm{p}<0.01)$. Me-1 and Pgi produced $D$ values close to zero (except in SFT and BR) coincident with the goodness of fit of proportions in all populations and the positive $D$ value for the Pgm gene only was significant ( $p<$ $0.05)$ in CSMM. Me-2 and Xdh, which yielded high negative $D$ values $(-0.268$ and 0.562 , respectively), did not have null alleles and the frequencies of all electromorphs were $>0.05$. Nevertheless, only $\mathrm{Me}-2$ was at equilibrium in SBD, SBPM and CLC populations, and CLC was also the only population which did not show departures from Hardy-Weinberg proportions for the $X d h$ gene. Selection and/or hidden variability could be a likely explanation for disequilibria of these genes. A. second possible explanation may be the finding that many electromorphs are known to harbour hidden variation that can be uncovered by varying the assay conditions (Rebordinos \& Pérez de la Vega 1990)

The amount of allele frequency variance as measured by $F_{\mathrm{ST}}$ was fairly small at most loci and the overall differentiation between populations was slight according to a mean $F_{\mathrm{ST}}$ of 0.031 . This value was on the same order of magnitude as that reported for other local population of bivalves (Buroker et al. 1979, Holborn et al. 1994). In the same way, for the oyster species Pinctada maxima the average $F_{\mathrm{ST}}$ value with 6 polymorphic enzymes from 5 areas which span a distance of $3400 \mathrm{~km}$ was 0.104 (Johnson \& Joll 1993). Notably higher values have been described for species with no planktonic development (Johnson \& Black 1991)

Acknowledgements. We thank J. M. Cantoral and l. Moreno for assistance in the collection of the samples. This study was supported by the Project UCA GRPRE-94/03 of the University of Cádiz.

\section{LITERATURE CITED}

Banks MA, Hedgecock D, Waters C (1993) Discrimination between closely related Pacific oyster species 1 Crassostrea) via mitochondrial DNA sequences cading for large subunit rRNA. Mol Mar Biol Biotechnol 2(3):129-136

Beaumont AR (1982) Variation in heterozygosity at two loci between year classes of a population of Chlamys opercularis (L.) from a Scottish sea-loch. Mar Biol Lett 3: 25-34

Beaumont AR, Beveridge CM, Budd MD (1983) Selection and heterozygosity within single famlies of the mussel Mytilus edulis (L.). Mar Biol Lett 4:151-161 
Buroker NE, Hershberger WK, Chew KK (1979) Population genetics of the Family Ostreidae. I. Intraspecific studies of Crassostrea gigas and Saccostrea commercialis. Mar Biol $54: 157-169$

Foltz DW (1986a) Null alleles as a possible cause of heterozygote deficiencies in the oyster Crassostrea virginica and other bivalves. Evolution 40:869-870

Foltz DW (1986b) Segregational and linkage studies of allozyme loci in pair crosses of the oyster Crassostrea virginica. Blochem Genet 24:941-956

Gaffney PM, Standish KA Jr (1.993) Hybridization among Crassostrea species: a review. Aquaculture 116:1-13

Hare MP, Karl SA, Avise JR (1996) Anonymous nuclear DNA markers in the American oyster and their implications for the heterozygote deficiency phenomenon in marine bivalves. Mol Biol Evol 13:334-345

Hedrick PW (1971) An approach to measuring geneitc similanty. Evolution 25:276-280

Hilbish JT, Koehn RK (1985) The physiological basis of natural selection at the Lap locus. Evolution 39(6) $1302-1317$

Holborn K, Johnson MS, Black R (1994) Population genetics of the corallivorous gastrupud Drupella cornus at Ningallo Reef, Western Australia. Coral Reefs 13:33-39

Johnson MS, Black R (1991) Genetic subdivision of the intertidal snai] (Bembicium vittatum) (Gastropoda: Litforinidae) varies with habitat in the Houtman Abrolhos Islands, Westen Austrâlia. I léredity $67: 20 j-213$

Johnson MS, Joll LM (1993) Genetic subdivision of the pearl oyster Pinctada maxima (Jameson, 1901) (Mollusca: Pteriidae) in northern Australia. Aust J Mar Freshwat Res 44: $519-526$

Koehn RK, Milkman R, Mitton JB (1976) Population genetics of marine pelecypods. IV. Selection, migration and genetic differentiation an the blue mussel Mytilus edulis. Evolution 30:2-32

Koehn RK, Newell R, Immermann F (1980) Maintenance of an aminopeptidase allele frequency line by natural selection. Proc Natl Acad Sci USA 77:5385-5389

Korringa P (1952) Recent advances in oyster biology. Q Rev Biol 27:266-308

Mallet AL, Zouros E, Gartner-Kepkay KE, Freeman KR, Dickie L.M (1985) Larval viability and heterozygote deficiency in populations of marne bivalves: evidence from pair matings of mussels. Mar Biol 87:165-172

Marteil L (1960) Ecologie des huitres du Mordihan, Ostrea

This article was submitted to the editor edulis Linné et Gryphaea angulata Lamarck. Rev Trav Inst Peches Marit 24:329-446

Nei M (1977) F-statıstics and analysis of gene diversity in subdivided populations. Ann Hum Genet 41:225-233

Nei $M$ (1978) Estimation of average heterozygosity and genetic distance from a small number of individuals. Genetics 89:583-590

Rebordinos L, Pérez de la Vega M (1987) The inheritance of seed peroxidases of wheat and rye: further data. Theor Appl Genet 74:767-772

Rebordinos L, Pérez de la Vega M (1990) Genetic variability of leaf esterases in Triticum aestivum L. $2 n=6 x=42$. Theor Appl Genet 79:673-678

Sarver SK, Katoh M, Foltz DW (1992) Apparent overdominance of enzyme specific activity in two marine bivalves. Genetica 85:231-239

Selander RK, Smith MH, Yang SY, Johnson WE, Gentry JB (1971) Biochemical polymorphism and systematics in the genus Peromyscus. I. Variation in the old-field mouse (Peromyscus polionotus). Stud Genet 6:49-90

Siegesmund HR (1985) Genetic studies of Gammarus. IV Selection component analysis of the Gpi and the Mpi loci in Cammarus oceanicus. Hereditas 102:241-250

Singh SM, Green RH (1984) Excess of allozyme homozygosity in marine molluscs and its possible biological significance. Malacologia 25:569--581.

Singh SM, Zouros E (1978) Genetic variation associated with growth rate in the American oyster (Crassostrea virginica). Evolution 32:34.2-353

Slatkin M (1987) Gene flow and the geographic structure of natural populations. Science 236:787-792

Sneath PHA, Sokal RR (1973) Numerical taxonomy. WH Freeman, San Francisco

Staton JL, Felder DL (1995) Genetic variation in populations of the ghost shrimp genus Callichirus (Crustacea: Decapoda: Thalassinoidea) in the western Atlantic and Gull of Mexico. Bull Mar Sci 56:523-536

Swofford DL, Selander RB (1981) BIOSYS-1: a Fortran program for the comprehensive analysis of electrophoretic data in population genetics and systematics. J Hered 72:281-283

Thiriot-Quiévreux C (1984) Analyse comparée des caryotypes d'Ostreidae (Bivalvia). Cah Biol Mar 25:407-418

Wright S (1943) Isolation by distance. Genetics 28:114-138

Zouros E, Romero-Dorey M, Mallet AL (1988) Heterozygosity and growth in marine bivalves: further data and possible explanations. Evolution 42:1332-1341

Manuscnpt received: March 25, 1997

Revised version accepted: June 4, 1997 